\title{
ARTICLE
}

\section{Temperature effect in time structure of light emission in crystalline scintillators}

\author{
Toshinori NAGASAKI*, Masahiko UEYAMA, Masakatsu YOSHIOKA, Katashi KIYOHARA, \\ Yusuke KOBA, Hiroki IWAMOTO, and Yusuke UOZUMI \\ Department of Applied Quantum Physics and Nuclear Engineering, Kyushu University, \\ 744, Motooka, Nishi-ku, Fukuoka 819-0395, Japan
}

\begin{abstract}
The time structure of light emission in crystalline scintillators, $\mathrm{NaI}(\mathrm{Tl}), \mathrm{GSO}(\mathrm{Ce})$ and $\mathrm{LYSO}(\mathrm{Ce})$ have been investigated in a high temperature region. Main experiments were made at extremely high-temperature by heavy-ion bombardment at relativistic energies. Supplemental experiments were made through the gamma-ray incidence at temperature of 27,40 , and $51^{\circ} \mathrm{C}$ with a conventional heater. The measured results were compared and discussed.
\end{abstract}

KEYWORDS: NaI(Tl), GSO(Ce), LYSO(Ce), time structure, decay curve, temperature dependence

\section{Introduction}

The light emission variations of crystalline scintillators have been a controversial subject for a long time. For instance, a great deal of effort has been made particularly on the scintillation efficiency of $\mathrm{NaI}(\mathrm{Tl})$ with bombardments by energetic heavy-ions at different stopping power $\mathrm{dE} / \mathrm{dx}$. Experimental results have shown that the scintillation efficiency of $\mathrm{NaI}(\mathrm{Tl})$ crystals to heavy-ions decreases with increasing $\mathrm{dE} / \mathrm{dx}$. The gross structure of their $\mathrm{dE} / \mathrm{dx}$ dependence has been discussed by using a universal curve of the scintillation efficiency.

It is also a long time subject that $\mathrm{NaI}(\mathrm{Tl})$ shows the temperature dependence of the light yield. This dependence has been discussed in the last decade on the shape and the amplitude of light pulses emitted from the $\mathrm{NaI}(\mathrm{Tl})$ and other scintillators for a given energy of the incident ionizing radiation. The typical tendency is seen in the slow component whose intensity increases with decreasing temperature. This tendency has been interpreted in terms of the self-trap exciton formation $^{1)}$.

From a view point to understand scintillation mechanism with heavy-ion bombardment, it is interesting to investigate the temperature dependence of the temperature dependence of the time profile. We firstly observe the time structure of light emission under different $\mathrm{dE} / \mathrm{dx}$ conditions. If the $\mathrm{dE} / \mathrm{dx}$ dependence is significant, a new development of particle-identification can be expected. The experiment is conducted at HIMAC of the National Institute for Radiological Sciences, Japan. Secondly, we observe the temperature dependence of gamma-ray excited scintillation time profile under various temperature conditions of scintillator at a laboratory of Kyushu University. By using a commercial heater, moderate high-temperature is reached.

The purpose of the present work is to observe the time structure of the light pulse of crystalline scintillators; $\mathrm{NaI}(\mathrm{Tl})$, $\mathrm{GSO}(\mathrm{Ce})$ and $\mathrm{LYSO}(\mathrm{Ce})$ with heavy-ion and gamma-ray incidence. To achieve accurate time-profile measurements a TOF-delayed-coincidence technique was applied.

*Corresponding Author, E-mail:nagasaki@nucl.kyushu-u.ac.jp

(C) Atomic Energy Society of Japan

\section{Experiments}

The experiments were carried out at the Heavy-Ion Medical Accelerator in Chiba (HIMAC) of NIRS and our laboratory. Both experiments used almost same measurement techniques that draw upon the Delayed-Coincidence Method ${ }^{2-3)}$. This method was developed by Bollinger and Thomas to measure the decay time profile of scintillators.

At HIMAC experiments, the beam energies were 230 $\mathrm{MeV} / \mathrm{u}$ for Helium ions, $290 \mathrm{MeV} / \mathrm{u}$ for Carbon ions, and $650 \mathrm{MeV} / \mathrm{u}$ for Argon ions. The entire set up was shown schematically in Fig. 1. The scintillators used were $\mathrm{NaI}(\mathrm{Tl})$, $\mathrm{GSO}(\mathrm{Ce})$, and LYSO $(\mathrm{Ce})$. These scintillators were of a cubic shape having a $50.8-\mathrm{mm}, 48-\mathrm{mm}$, and $20-\mathrm{mm}$ edge length, respectively. In Table 1 are listed the parameters of used scintillator at present experiments. All six surfaces of these scintillators were mirror polished. One of the faces was contacted tightly to a photomultiplier tube (PMT) Hamamatsu R580, from which the time of radiation incidence was provided. The opposite side of this was wrapped with aluminum foil that has a small hole at its center, where a single photon is allowed to reach at a PMT (R6427) placed apart from the crystal to pick up the time information of scintillation. The other four surfaces were wrapped completely with aluminum foils for scintillation light reflection.

Table 1

The parameter of scintillators used at present experiments.

\begin{tabular}{lccc}
\hline Scinillator & $\mathrm{NaI}(\mathrm{Tl})$ & $\mathrm{GSO}(\mathrm{Ce})$ & $\mathrm{LYSO}(\mathrm{Tl})$ \\
\hline Density $\left(\mathrm{g} / \mathrm{cm}^{3}\right)$ & 3.76 & 6.71 & 7.1 \\
Decay time $(\mathrm{nsec})^{*}$ & 230 & 56 (fast) & 41 \\
& \multicolumn{3}{c}{400 (slow) } \\
Light output (arb.) & 100 & 20 & 40 \\
Size (mm) & 50.8 & 43 (HIMAC) & 20 \\
& \multicolumn{3}{c}{$\phi 25 \times 5$ (our lab. ) } \\
\hline
\end{tabular}

*Measured at room temperature 


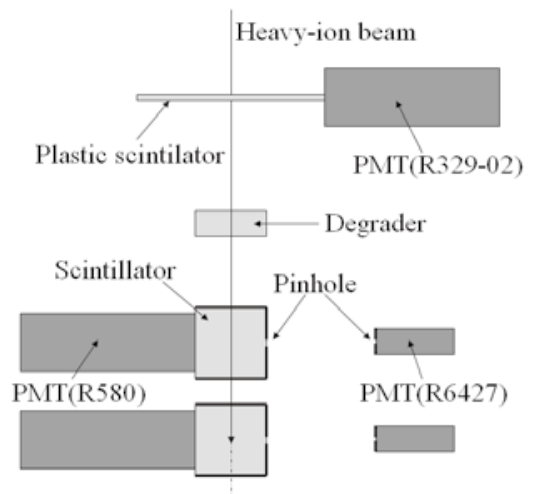

Fig. 1 Set up of the experiment at the HIMAC

Table 2

The expected specific energy losses calculated with the Bethe formula at the center of scintillator

\begin{tabular}{llccc}
\hline & & \multicolumn{3}{c}{$\mathrm{dE} / \mathrm{dx}\left(\mathrm{MeVcm}^{2} / \mathrm{g}\right)$} \\
& & ${ }^{40} \mathrm{Ar}$ & ${ }^{12} \mathrm{C}$ & ${ }^{4} \mathrm{He}$ \\
\hline $\mathrm{NaI}(\mathrm{Tl})$ & $\begin{array}{c}\text { Penetrate } \\
\text { Stop }\end{array}$ & 600 & 100 & 10 \\
& & 44000 & 7000 & 940 \\
$\mathrm{GSO}(\mathrm{Ce})$ & $\begin{array}{l}\text { Penetrate } \\
\end{array}$ & 600 & 100 & 10 \\
& Stop & 34000 & - & 940 \\
$\mathrm{LYSO}(\mathrm{Ce})$ & Penetrate & 640 & 100 & 10 \\
& Stop & 38000 & 4900 & 770 \\
\hline
\end{tabular}

In order to choose suitable particle energies, degraders were placed in front of the scintillation detectors, to make particles stop at the center of the second crystal. The energy of penetrate beam was measured with the front crystal and the stop beam energy was measured with the rear one. In Table 2 are listed the expected specific energy losses calculated with the Bethe formula.

The set up of experiment which was carried out at our laboratory is shown schematically in Fig. 2. The ${ }^{137} \mathrm{Ce}$ gamma-source was placed under the PMT(R580), and the $\mathrm{GSO}(\mathrm{Ce})$ scintillator $(\phi 25 \times 5 \mathrm{~mm})$ was optically coupled to this PMT. The opposite side of this scintillator surface was wrapped with aluminum foil that has a diameter of $1.0-\mathrm{cm}$ hole, and the other surface was mirror polished and wrapped with aluminum foil. The temperature was controlled by a heater shown schematically in Fig. 2 and monitored to be stable within $\sim 0.1^{\circ} \mathrm{C}$ preset value by a small thermometer attached on the scintillator. The range of temperature at this experiment were from room temperature to about $50^{\circ} \mathrm{C}$.

Both experiments, charges of linear signals from PMT were digitized with CAMAC ADCs and taken by a PC system.

\section{Results and discussion}

The representative results from present experiment at HIMAC are displayed in histogram forms in Figs. 3-5 with the ${ }^{137} \mathrm{Cs}$ gamma-ray source result at room

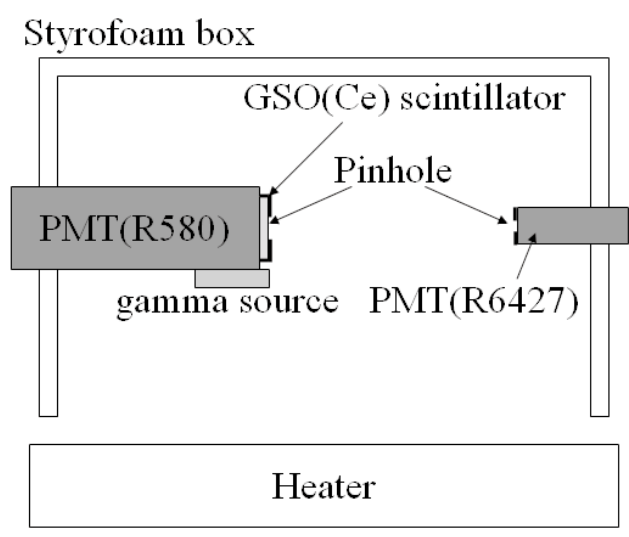

Fig. 2 Set up of the experiment at a laboratory of Kyusyu University

temperature. Each one is normalized at the peak value to make the comparison easier and clearer.

In Fig. 3 is shown a decay curve for ${ }^{4} \mathrm{He}$ incidence at an energy $230 \mathrm{MeV} / \mathrm{u}$, which stopped at the center of $\mathrm{NaI}(\mathrm{Tl})$ scintillator(He_stop) and that for gamma-ray(gamma-ray). The comparison of two, the decay curve of He_stop is smaller than that of gamma-ray from $30 \mathrm{nsec}$ to $1000 \mathrm{nsec}$. In Fig. 6 is shown the part which was confirmed this difference for ${ }^{40} \mathrm{Ar},{ }^{12} \mathrm{C}$, and ${ }^{4} \mathrm{He}$ incidence data of present works. Except for the decay curve of He_penetrate, all of decay curves are almost same in shape. By comparison the data and the value of Table 2, the decay curve of $\mathrm{NaI}(\mathrm{Tl})$ scintillator depends on the $\mathrm{dE} / \mathrm{dx}$ and the curve is changing at $100\left(\mathrm{MeVcm}^{2} / \mathrm{g}\right) \mathrm{dE} / \mathrm{dx}$ or less.

In Fig. 4 is shown a decay curve for ${ }^{40} \mathrm{Ar}$ beam of 650 $\mathrm{MeV} / \mathrm{u}$, which penetrates at the center of $\mathrm{GSO}(\mathrm{Ce})$ scintillator(Ar_penetrate) and a decay curve for gamma-ray(gamma-ray). The relative intensity of Ar_penetrate is larger than that of gamma-ray from 300 to 1000 nsec. This range is enlarged and shown in Fig. 7

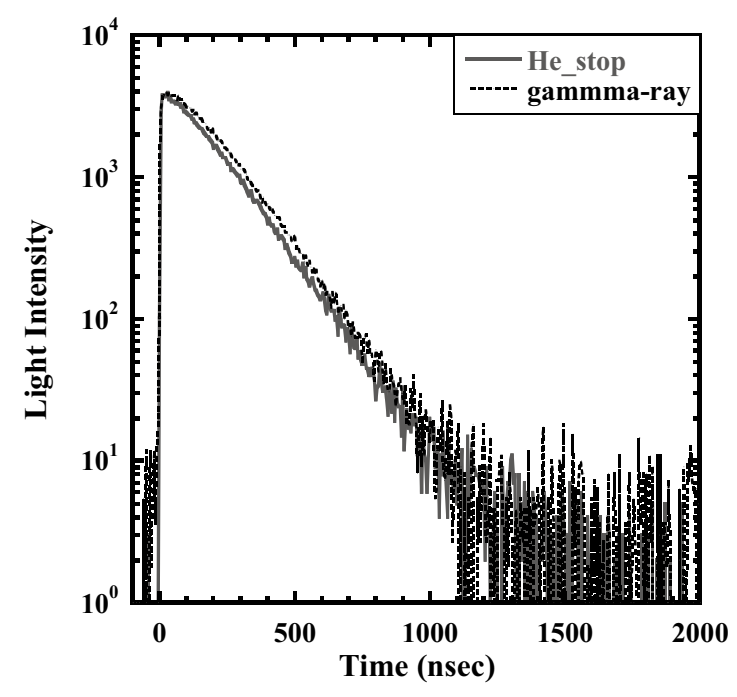

Fig. 3 Typical time structure of scintillation from $\mathrm{NaI}(\mathrm{Tl})$ for He_stop and gamma-incidence. 


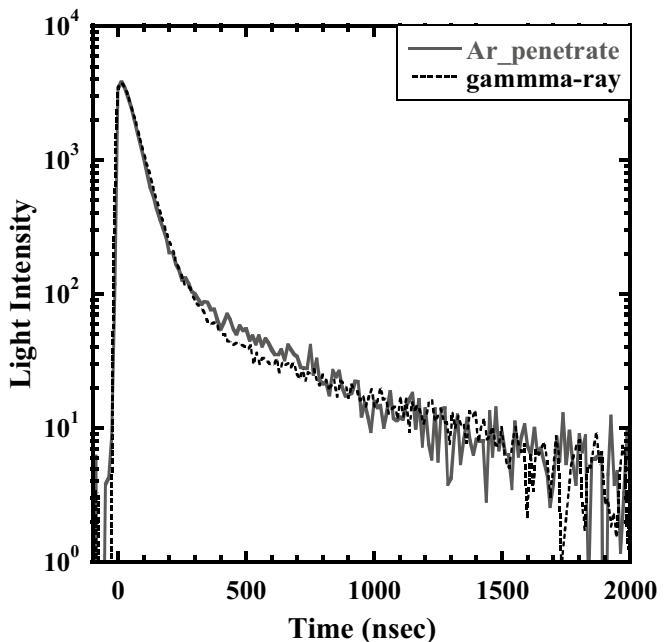

Fig. 4 Typical time structure of scintillation from $\mathrm{GSO}(\mathrm{Ce})$ for Ar_penetrate and gamma-incidence.

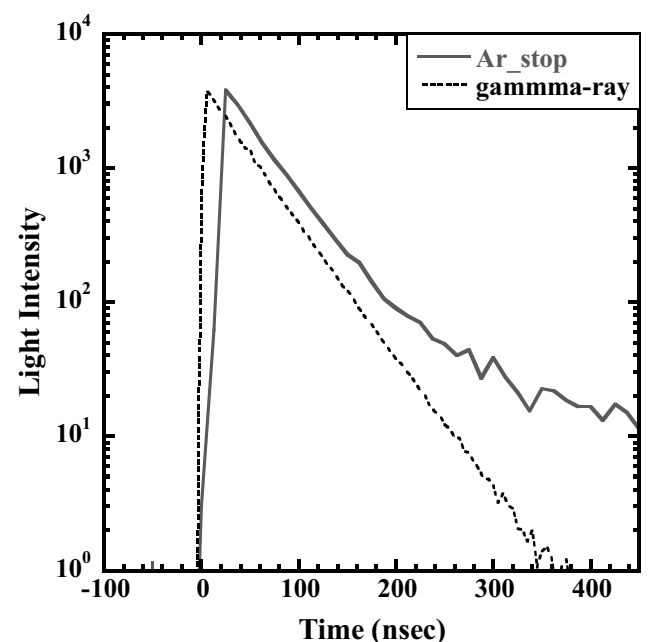

Fig. 5 Typical time structure of scintillation from $\operatorname{LYSO}(\mathrm{Ce})$ for Ar_stop and gamma-incidence.

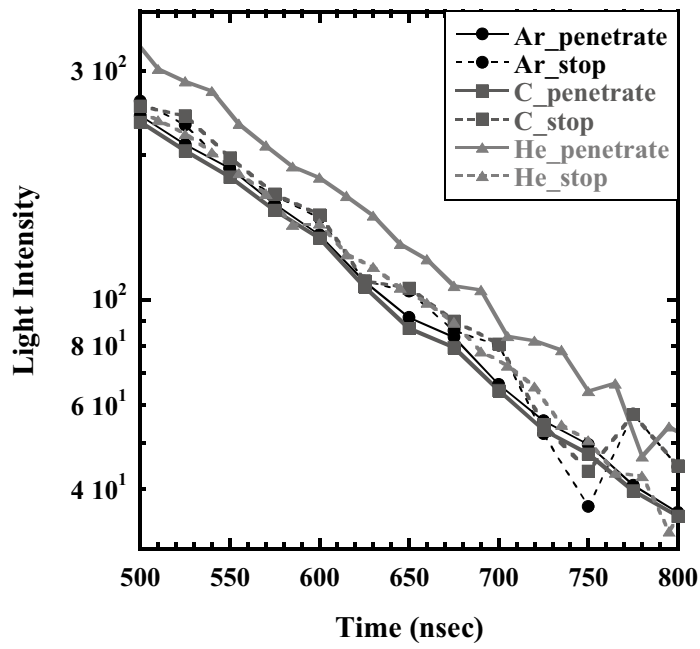

Fig. 6 Measured time structure of scintillation from $\mathrm{NaI}(\mathrm{Tl})$ in a time range between 500 and $800 \mathrm{nsec}$

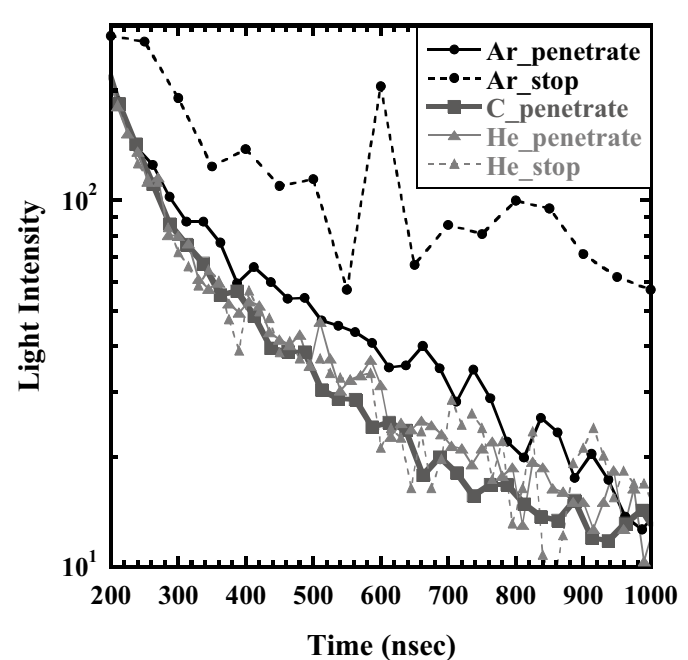

Fig. 7 Measured time structure of scintillation from $\mathrm{GSO}(\mathrm{Ce})$ in a time range between 200 and 1000 nsec.

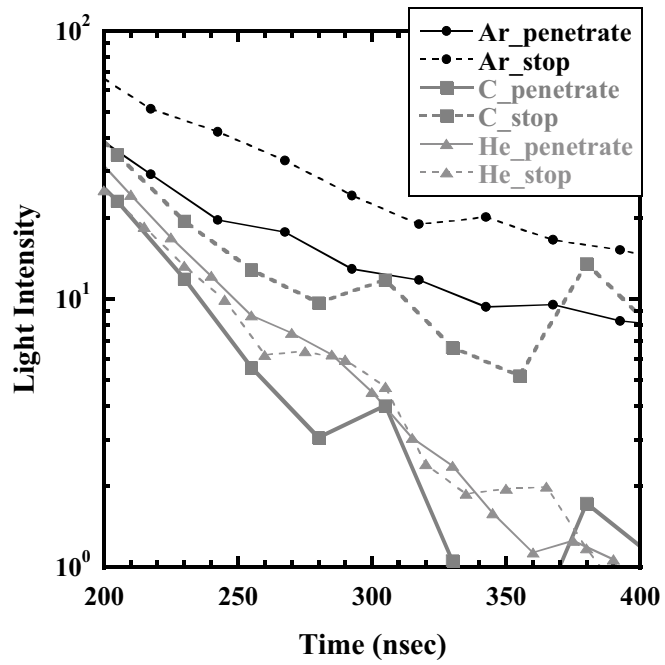

Fig. 8 Measured time structure of scintillation from $\operatorname{LYSO}(\mathrm{Ce})$ between 200 and $400 \mathrm{nsec}$

for all experimental data. The relative intensity for Ar_stop is the largest in this slow component range and that for Ar_penetrate is the second. The others, He_penetrate, He_stop and C_penetrate are of almost the same in this time range. As shown in Table 2, the $\mathrm{dE} / \mathrm{dx}$ value of $\mathrm{He}$ stop is larger than that of Ar penetrate. The present result should imply that the decay constant depends on not only $\mathrm{dE} / \mathrm{dx}$. Actually, the scintillation efficiency is widely known to depend on the particle identity as well as $\mathrm{dE} / \mathrm{dx}$.

In Fig.5 is shown a decay curve for ${ }^{40} \mathrm{Ar}$ beam of energy $650 \mathrm{MeV} / \mathrm{u}$, which stopped at the center of LYSO(Ce) scintillator (Ar_stop) and that for gamma-ray (gamma-ray). Rather large differences can be seen between these two cases. The rise time for the Ar_stop is longer than that for gamma-ray. A single decay component is observed for gamma-rays, as known widely; however, the Ar_stop result reveals two decay 
components. Fig. 8 shows the enlarged part of decay curves for all experimental data. In this time range, the relative intensity of Ar_stop is largest. Those of Ar_penetrate and $\mathrm{C}$ stop are the next. And the others, $\mathrm{He}$ penetrate, He_stop, and $\mathrm{C}$ penetrate follow with similarity in this range. Along with $\mathrm{GSO}(\mathrm{Ce})$ scintillator, the value of $\mathrm{dE} / \mathrm{dx}$ of $\mathrm{He}$ stop is larger than that for Ar_penetrate in the $\operatorname{LYSO}(\overline{\mathrm{Ce}})$ scintillator. But the value of the decay curve for He_stop is lower than that for Ar_penetrate. We expected that the decay curve of these scintillators depends on not only $\mathrm{dE} / \mathrm{dx}$ of incident particle but also the characteristics of the incident particle.

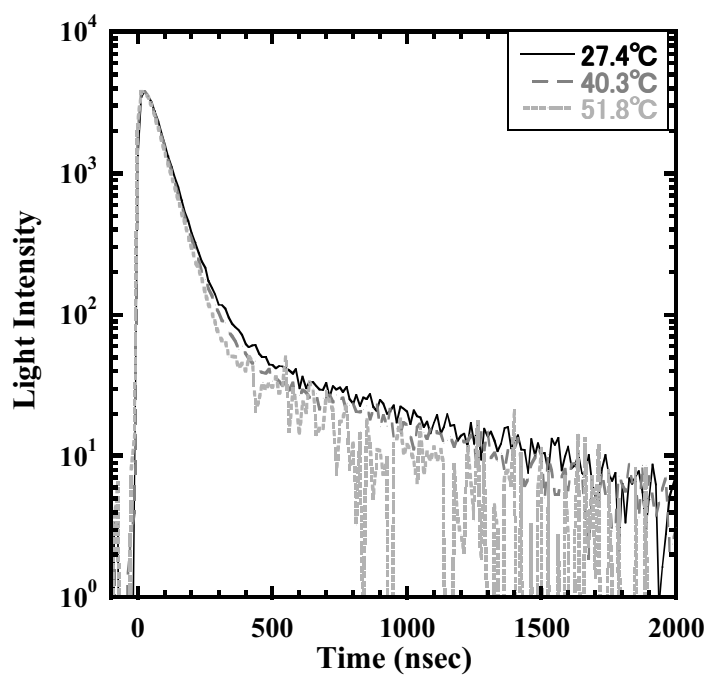

Fig. 9 Measured time structure of scintillation from $\mathrm{GSO}(\mathrm{Ce})$ at $27.4^{\circ} \mathrm{C}, 40.3^{\circ} \mathrm{C}$, and $51.8^{\circ} \mathrm{C}$

The temperature dependence of the gamma-ray excited scintillation time profile of $\mathrm{GSO}(\mathrm{Ce})$ scintillator, which was measured at a laboratory of Kyusyu University, is shown in Fig. 9, where the comparison is made between temperatures of $27.4^{\circ} \mathrm{C}, 40.3^{\circ} \mathrm{C}$, and $51.8^{\circ} \mathrm{C}$. As the temperature of the $\mathrm{GSO}(\mathrm{Ce})$ scintillator increases from room temperature, the slow component appears to be suppressed. No difference is seen in the fast component. It is the opposite tendency compared with that of heavy-ion incidence providing different $\mathrm{dE} / \mathrm{dx}$ conditions, if one considers the larger $\mathrm{dE} / \mathrm{dx}$ corresponds to the higher temperature.

\section{Conclusion}

We observed the time structure of light emission of inorganic scintillator $\mathrm{NaI}(\mathrm{Tl}), \mathrm{GSO}(\mathrm{Ce})$, and $\mathrm{LYSO}(\mathrm{Ce})$ under different conditions. The $\mathrm{dE} / \mathrm{dx}$ dependence was investigated at HIMAC by using heavy-ion beams. The temperature dependence was also studied for gamma-ray incidence at temperature $27^{\circ} \mathrm{C}, 40^{\circ} \mathrm{C}$ and $51^{\circ} \mathrm{C}$.

The scintillation time profiles of all crystals have appeared to change with both $\mathrm{dE} / \mathrm{dx}$ and temperature. From the pulse shape analyses, we have found that these results cannot be interpreted by the decay time change of a single component, but they should be attributed to the change of the slow component intensity. The first component is insensitive to $\mathrm{dE} / \mathrm{dx}$ and temperature.

In the case of the $\mathrm{GSO}(\mathrm{Ce})$ scintillator for instance, the results of ion-bombardment experiments indicate that the slow component becomes smaller with $\mathrm{dE} / \mathrm{dx}$ larger. The temperature dependence of the slow component has been found to decrease with increasing temperature. These results are rather unreasonable because the $\mathrm{dE} / \mathrm{dx}$ dependence would be similar to the temperature dependence, because a local heating is expected around the ion track. It should be noticed that other crystals show different dependences from each other. The variation of absolute scintillation yields cannot be discussed, since we measured just the relative scintillation intensity.

\section{Acknowledgment}

Authors gratefully acknowledge Dr. M. Imamura for useful information and discussion. The present study was conducted under the HIMAC program number $20 \mathrm{P} 239$.

\section{References}

1) B. S. Alexandrov, K. D. Ianakiev, P. B. Littlewood, "Branching transport model of $\mathrm{NaI}(\mathrm{Tl})$ alkali-halide scintillator," Nuclear Instruments and Methods in Physics Research Section A, 586, 432 (2008).

2) L. M. Bollinger, G. E. Thomas, "Measurement of the Time Dependence of Scintillation Intensity by a Delayed-Coincidence Method," The Review of Scientific Instruments, 32, 1044 (1961).

3) N. Tsuchida, M. Ikeda, T. Kamae, et al., "Temperature dependence of gamma-ray excited scintillation time profile and light yield of GSO, YSO, YAP and BGO," Nuclear Instruments and Methods in Physics Research Section A, 385, 290 (1997)

4) G. F. Knoll, "Radiation Detection and Measurement third edition," 2001 